Case

Report

\title{
A Recurrent Vagal Schwannoma in the Middle Mediastinum after Surgical Enucleation
}

\author{
Kishu Fujita, MD, ${ }^{1}$ Kuniki Nakashima, MD,${ }^{1}$ Hisao Kumakura, MD, ${ }^{2}$
}

and Kazutomo Minami, MD ${ }^{1}$

\begin{abstract}
A 50-year-old man underwent repeat surgery for a benign vagal schwannoma in the middle mediastinum. He had undergone tumor enucleation at another hospital 4 months before presentation. The tumor $(99 \times 88 \times 76 \mathrm{~mm})$ was located in the aortopulmonary window and arose from the left vagus nerve. It had been enucleated, leaving its sheath behind to preserve the nerve. Imaging studies showed tumor regrowth without distant metastasis, and the tumor was extirpated along with the involved nerve during cardiopulmonary bypass. There was no nerve dysfunction, recurrence, or metastasis 6 months after the operation. A benign vagal schwannoma can be excised with nerve transection or enucleated without nerve transection. The present case suggests that a vagal mediastinal schwannoma should be extirpated along with the nerve because insufficient enucleation might lead to tumor regrowth.
\end{abstract}

Keywords: mediastinal neoplasms, neurilemmoma, vagus nerve diseases, postoperative complications

\section{Introduction}

Schwannoma is the most common intrathoracic neurogenic tumor, typically arising along an intercostal nerve or a sympathetic chain within the posterior mediastinum. $^{1-3)}$ It is basically benign, but malignant degeneration has been reported in $10 \%$ of cases. ${ }^{4,5}$ Schwannoma is nonresponsive to radiation or chemotherapy and should thus be resected, not only to prevent further

${ }^{1}$ Department of Cardiovascular Surgery, Kitakanto Jyunkanki Hospital, Gunma, Japan

${ }^{2}$ Department of Internal Medicine, Kitakanto Jyunkanki Hospital, Gunma, Japan

Received: August 9, 2012; Accepted: November 7, 2012

Corresponding author: Kishu Fujita, MD. Kitakanto Jyunkanki Hospital, 740 Shimohakoda, Hokkitsumachi, Shibukawa, Gunma 377-0061, Japan

Email: kishufujita@ccj.or.jp

(C)2014 The Editorial Committee of Annals of Thoracic and Car-

diovascular Surgery. All rights reserved. growth and compression of adjacent structures, but also to confirm the nature of the lesion. ${ }^{1,6)}$ It can be cured by surgery, and recurrence is unusual, except in patients with neurofibromatosis. ${ }^{1,2}$

\section{Case Report}

A 50-year-old man presented with progressive dyspnea and palpitation persisting for 1 month. The patient had undergone surgery for a benign vagal schwannoma in the middle mediastinum. The operation was performed via a median sternotomy at another hospital 4 months previously. The tumor, measuring $99 \times 88 \times 76 \mathrm{~mm}$, was located inferior to the aortic arch, posterior to the main pulmonary arteries, superior to the left atrium, and anterior to the main bronchi (Fig. 1A). It was encapsulated and apparently arose from the left vagus nerve distal to the takeoff of the left recurrent nerve. It adhered to the adjacent structures and was inseparable from the involved nerve. Therefore, the tumor could nothing but be enucleated, 

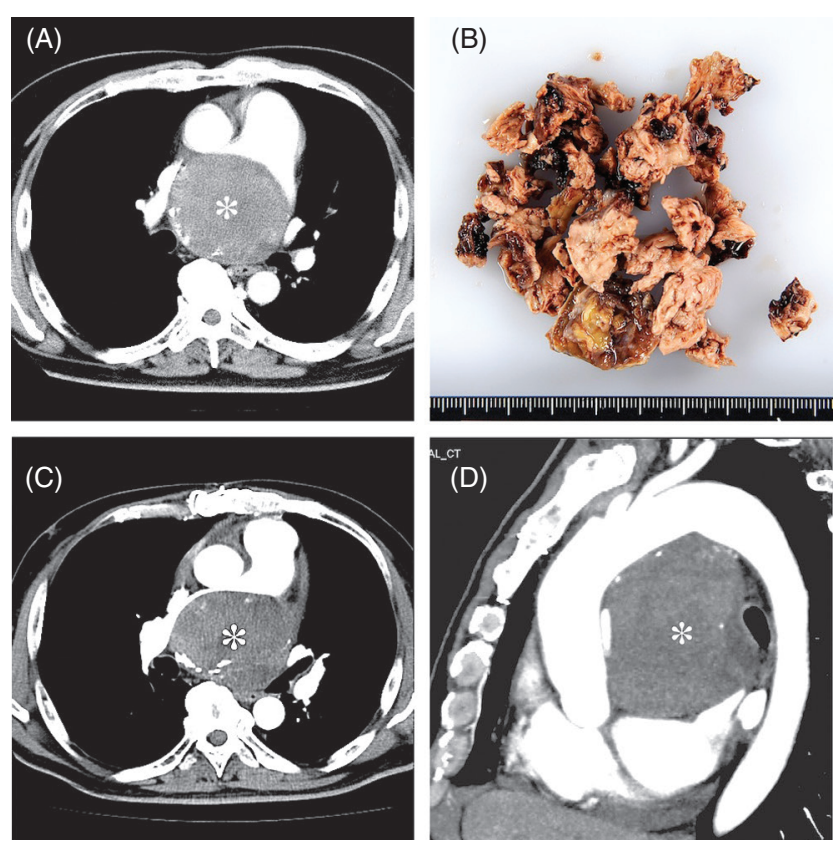

Fig. 1 (A) Chest CT imaging before the first operation. The tumor is marked with an asterisk. (B) Photograph of the tumor enucleated in pieces at the previous surgery. (C) Transverse section, and (D) Sagittal section of the chest CT imaging at our presentation showing the tumor (asterisk) similar in size and location as that before surgery on (A).

which means only its contents had been removed partially piece by piece, leaving its sheath behind to avoid nerve transection (Fig. 1B). The immunohistopathological diagnosis was a benign schwannoma. The postoperative course was uneventful, and the patient remained asymptomatic for 3 months.

On admission, the patient was not in distress, with blood pressure of $130 / 70 \mathrm{mmHg}$, heart rate of 72 beats/min, and the body temperature of $36.6^{\circ} \mathrm{C}$. Physical examination was unremarkable, with no clinical signs suggesting von Recklinghausen's neurofibromatosis. Electrocardiography and blood laboratory tests were unremarkable. Serum levels of CEA, CA 19-9, CA 125, AFP, HCG, and NSE were normal. Chest roentgenography showed only a slightly enlarged mediastinal shadow. Transthoracic echocardiography revealed a $70 \times 69 \mathrm{~mm}$ mediastinal tumor on the roof of the left atrium. The tumor compressed both pulmonary arteries, with no echocardiographic pulmonary artery stenosis or hypertension. The function of the left ventricle was preserved. Enhanced computed tomography (CT) and magnetic resonance imaging (MRI) of the chest revealed regrowth of the tumor. The tumor severely compressed adjacent tis-
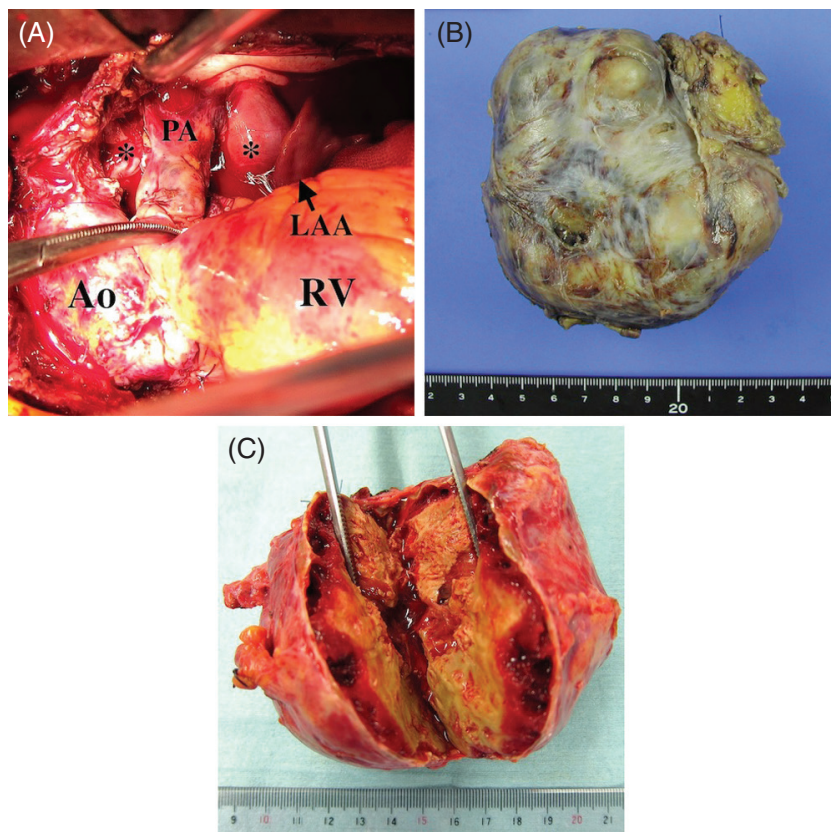

Fig. 2 Photographs of the tumor weighing $250 \mathrm{~g}$ and measuring $85 \times 85 \times 55 \mathrm{~mm}$. (A) An intraoperative picture from the surgeon's point of view. The tumor is marked with asterisks. Ao: ascending aorta; RV: right ventricle; PA: left pulmonary artery; LAA: left atrial auricle. (B) Gross appearance on the posterior side of the tumor. (C) Transected section of the tumor showing an encapsulated solid mass with a yellowish cut surface and internal hemorrahage.

sues and measured $90 \times 86 \times 72 \mathrm{~mm}$. The size and location were similar to those before the previous surgery (Fig. 1C and 1D). Whole-body enhanced CT showed no other abnormalities. Positron emission tomography with fluorine-18 fluorodeoxyglucose showed a slight uptake and only within the tumor limb. No abnormal uptake inside the tumor or evidence of distant metastases was detected. The tumor most likely caused the patient's symptoms by compressing the surrounding structures. Thus, reoperation was planned.

Exposure was obtained through a repeat median sternotomy. Difficulty in adequately and safely exposuring the tumor, combined with its size, location, and adhesion, required cardiopulmonary bypass and cardioplegic arrest. The entire encapsulated tumor was completely and successfully freed from its adjacent tissues by retracting the heart cephalad and transiently disconnecting the main pulmonary artery. The involved left vagus nerve was inseparable from the tumor and was, therefore, excised in conjunction with the tumor sheath (Fig. 2). The disconnected main pulmonary artery was sutured and reconnected. There was no evidence of 
(A)

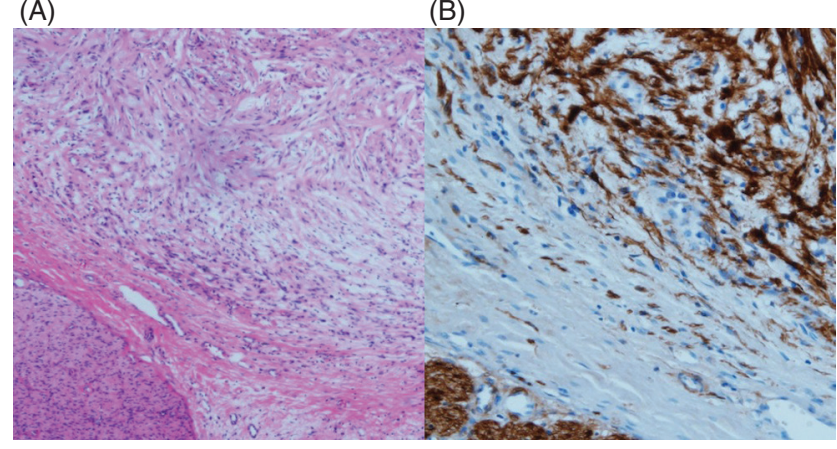

Fig. 3 Photomicrographs of the tumor. (A) Tumor cells composed of spindle cells arranged in whorl formation with no abnormal mitosis (hematoxylin and eosin, $\times 40$ magnification). (B) Tumor cells showing positive immunohistochemical stainings for S-100 (×100 magnification).

myocardial invasion or mediastinal lymphadenopathy. Final histopathological examination of the resected tumor confirmed a benign schwannoma with clean margins and positive immunohistochemical staining for S-100 (Fig. 3).

The postoperative course was uneventful. Hoarseness, dysphagia, or aspiration did not develop. Paroxysmal atrial fibrillation occurred sporadically without hemodynamic deterioration and was controlled by antiarrhythmic agents. The patient has remained asymptomatic for 6 months after the operation. Arrhythmia has not recurred since 4 weeks after surgery. Neither a local recurrence nor distant metastases were detected on whole body enhanced CT.

\section{Discussion}

Vagal schwannoma, which arises from the vagus nerve in the middle mediastinum, is extremely rare, accounting for $2 \%$ of all intrathoracic schwannomas. ${ }^{4)}$ To our knowledge, approximately 70 cases of vagal schwannoma have been documented to date. However, recurrence after surgery has not been reported. ${ }^{1-4,6-8)}$

How to manage the involved vagus nerve and avoid postoperative nerve dysfunction is a matter of debate. Whether the involved nerve should be sacrificed to ensure complete tumor excision or be preserved by enucleating the tumor and preserving at least part of its sheath to avoid nerve dysfunction remains controversial. Operative procedures and subsequent neurological outcomes have been reported for 11 patients.

Even after tumor enucleation, vocal cord paralyses occurred in 2 cases. In 1 patient, the tumor was located superior to the left recurrent nerve, and in the other, it was located at the bifurcation of the left recurrent nerve. ${ }^{1,6)}$ In contrast, no neurologic complication occurred in a patient in whom the left vagus nerve was sacrificed inferior to the left recurrent nerve. ${ }^{1)}$ In the other 6 cases, hoarseness developed after nerve transection. ${ }^{2,47}$ However, no other neurologic disability (e.g., dysphagia or aspiration) was reported, although the left recurrent nerves were directly sacrificed in 5 patients, and the vagus nerve was transected proximally to the left recurrent nerve in the remaining patient. Hoarseness resolved in 4 of these patients: Spontaneously in 2 and after otholaryngological procedures in 2 . The right vagus nerves were amputated without subsequent neurologic complications in the remaining 2 patients. ${ }^{3,8)}$

Sacrificing the vagus nerve in surgery for vagal schwannoma does not cause serious neural dysfunction affecting the heart, bronchial muscles, or gastrointestinal tract, suggesting that compensatory changes occur. ${ }^{1,2)}$ The paroxysmal atrial fibrilation in our patient might have been related to the nerve transection, but subsided after several months. Hoarseness can occur, but its incidence might depend on the tumor location rather than the operative procedure. ${ }^{2)}$ Moreover, hoarseness unaccompanied by dysphagia or aspiration leads to no serious sequelae and can be transient or managed otolaryngologically.

\section{Conclusion}

Our experience suggests that a benign vagal schwannoma can recur without malignant transformation after tumor enucleation. We believe that a middle mediastinal vagal schwannoma should be totally extirpated in conjunction with its sheath and the involved nerve. The procedure should be performed during cardiopulmonary bypass as required.

\section{Disclosure Statement}

We declare that we have no conflicts of interest to disclose.

\section{References}

1) Dabir RR, Piccione W, Kittle CF. Intrathoracic tumors of the vagus nerve. Ann Thorac Surg 1990; 50: 494-7.

2) Davis CJ, Butchart EG, Gibbs AR. Neurilemmoma of the intrathoracic vagus nerve. Eur Respir J 1991; 4: 508-10. 
3) Singer RL. Thoracoscopic excision of a malignant schwannoma of the intrathoracic vagus nerve. Ann Thorac Surg 1995; 59: 1586-7.

4) Sugio $K$, Inoue $T$, Inoue $K$, et al. Neurogenic tumors of the mediastinum originated from the vagus nerve. Eur J Surg Oncol 1995; 21: 214-6.

5) Odell DD, Macke RA, O'Shea MA. Clamshell thoracotomy: a unique approach to a massive intrathoracic schwannoma. Ann Thorac Surg 2011; 91: 298-301.
6) Sasaki K, Kohno T, Mun M, et al. Thoracoscopic removal of middle mediastinal schwannoma originating from recurrent nerve. Thorac Cardiovasc Surg 2008; 56: 375-7.

7) Shirakusa T, Tsutsui M, Montonaga R, et al. Intrathoracic tumors arising from the vagus nerve. Scand J Thorac Cardiovasc Surg 1989; 23: 173-5.

8) Heitmiller RF, Labs JD, Lipsett PA. Vagal schwannoma. Ann Thorac Surg 1990; 50: 811-3. 